\title{
Remodeling of energy metabolism by a ketone body and medium-chain fatty acid suppressed the proliferation of CT26 mouse colon cancer cells
}

\author{
YUI KADOCHI, SHIORI MORI, RINA FUJIWARA-TANI, YI LUO, YUKIKO NISHIGUCHI, \\ SHINGO KISHI, KIYOMU FUJI, HITOSHI OHMORI and HIROKI KUNIYASU \\ Department of Molecular Pathology, Nara Medical University, Kashihara, Nara 634-8521, Japan
}

Received November 12, 2016; Accepted March 13, 2017

DOI: $10.3892 / \mathrm{ol} .2017 .6195$

\begin{abstract}
Normal and cancerous cells are suggested to have differential utilization of fatty acids and ketone bodies, which could be exploited in cancer therapy. The present study examined the effect of 3-hydroxybutyric acid (3-HBA), which is a ketone body generating acetyl-CoA, and lauric acid (LAA, C12:0), which is a medium-chain saturated fatty acid translocated to mitochondria in a carnitine-independent manner to produce acetyl-CoA, on the energy metabolism of mouse CT26 colon cancer cells. In CT26 cells expressing 3-HBA and LAA transporters, 3-HBA and LAA reduced cell proliferation, mitochondrial volume and lactate production, and increased oxidative stress, particularly in low-glucose conditions. Concurrent treatment with 3-HBA and LAA under glucose starvation had a synergistic effect on cell growth inhibition. In addition, LAA and LAA + 3-HBA promoted an imbalance in the expression of enzymes in the electron transport chain. These findings suggested that treatment with 3-HBA and/or LAA during glucose starvation may reprogram energy metabolism and decrease the proliferation of cancer cells.
\end{abstract}

\section{Introduction}

The difference in energy production between cancer cells and normal cells is considered to be one of the available targets for the treatment of cancer (1). Unlike normal cells, cancer cells obtain energy mostly via glycolysis, while mitochondrial oxidative phosphorylation is reduced (2). As tumor cells actively proliferate, they require a constant energy supply and it has been postulated that they switch to aerobic glycolysis

Correspondence to: Professor Hiroki Kuniyasu, Department of Molecular Pathology, Nara Medical University, 840 Shijo-cho, Kashihara, Nara 634-8521, Japan

E-mail: cooninh@zb4.so-net.ne.jp

Key words: 3-hydroxybutiryic acid, lauric acid, mitochondria, oxidative stress characterized by rapid ATP production, which is known as the Warburg effect (2). It is hypothesized that the purpose of the Warburg effect is to avoid the excessive generation of reactive oxygen species (ROS) during oxidative phosphorylation in mitochondria, and the subsequent oxidative stress that causes cell damage $(1,3)$. The differences in the energy metabolism between tumor and normal cells have previously been exploited in cancer treatment, and the efficacy of a number of currently used chemotherapeutic agents, including methotrexate, depends on ROS production (3).

Excessive oxidative stress can be generated by forcing cancer cells to produce energy via mitochondrial oxidation, which is expected to cause anticancer effects without injury to benign cells, as for the latter it is a normal metabolic process (4). A previous study indicated that ketone bodies and medium-chain fatty acids (MCFAs) may be used as a tool to induce cancer cells to switch between glycolysis and oxidative phosphorylation for energy production, indicating that ketogenic and MCFA-enriched diets may be beneficial in cancer therapy (5).

In mitochondria, fatty acids are metabolized to acetyl-CoA via $\beta$-oxidation, which can be diverted to the formation of ketone bodies under the conditions of glucose starvation $(5,6)$. $\beta$-hydroxybutyric acid ( $\beta$-HBA, also termed 3-HBA) is one of the three ketone bodies generated from fatty acids; it has been revealed that 3-HBA can be used for energy production in mitochondria through the tricarboxylic acid cycle, where it is converted into acetoacetic acid and then to acetyl-CoA $(7,8)$. Ketogenic diets rich in medium-chain triglycerides demonstrated inhibitory effects on cancer growth, and it was observed that prostate cancer cells had lower ability to utilize dietary fatty acids compared with normal prostate cells, indicating possible therapeutic potential. Lauric acid (LAA) is an MCFA with an aliphatic tail of 12 carbons, which, contrary to long-chain fatty acids (LCFAs), can be transported to the mitochondrial intermembrane space directly without the carnitine shuttle (9). Therefore, LAA is utilized through $\beta$-oxidation with higher efficacy than LCFAs (10). The present study examined the effect of 3-HBA and LAA on cancer cell proliferation, oxidative stress and stemness, with the aim of providing mechanistic insights into a potentially therapeutic effect of ketogenic diets for patients with cancer. 


\section{Materials and methods}

Cell culture and treatment. The mouse CT26 colon cancer cell line was provided by Professor I. J. Fidler (MD Anderson Cancer Center, Texas University, Austin, TX, USA). Cells were cultured in Dulbecco's modified Eagle's medium containing high glucose $(450 \mathrm{mg} / \mathrm{dl})$, low glucose $(100 \mathrm{mg} / \mathrm{dl})$ or no glucose (Wako Pure Chemical Industries, Ltd., Osaka, Japan), and supplemented with $10 \%$ fetal bovine serum (Sigma-Aldrich; Merck KGaA, Darmstadt, Germany) at $37^{\circ} \mathrm{C}$ in $5 \% \mathrm{CO}_{2}$. Cells were treated with $3-\mathrm{HBA}(1 \mathrm{mM}$; Sigma-Aldrich; Merck KGaA) and/or LAA (1.5 mM; Wako Pure Chemical Industries, Ltd.) for $24 \mathrm{~h}$ at $37^{\circ} \mathrm{C}$, and analyzed for viability using a tetrazolium dye (MTT; Sigma-Aldrich; Merck KGaA) assay, as previously described (11). 3-HBA was solubilized using PBS at room temperature. LAA was solubilized in $60^{\circ} \mathrm{C}$ PBS and kept at $37^{\circ} \mathrm{C}$ until use. All procedures were performed in accordance with the Ethical Guidelines for Human Genome/Gene Research enacted by the Japanese Government, which was approved by the Ethics Committee of Nara Medical University (approval no., 937-1).

Mitochondrial volume was assessed by staining mitochondria with MitoGreen by adding MitoGreen-dimethyl sulfoxide solution $(200 \mu \mathrm{M})$ to cultured cells $(50 \mathrm{nM}$ at working concentration) for $1 \mathrm{~h}$ at $37^{\circ} \mathrm{C}$ (Takara Bio, Inc., Otsu, Japan). Cell images were captured under a fluorescence microscope equipped with a camera (Leica Microsystems, Inc., Buffalo Grove, IL, USA), and fluorescence was evaluated in the images using Photoshop Image Analyzer (CS2; version 9.0.2; Adobe Systems, Inc., San Jose, CA, USA). Alkaline phosphatase (ALP) activity was determined using a commercial kit according to the manufacturer's protocol (LabAssay ALP; catalog no., 291-58601; Wako Pure Chemical Industries, Ltd.). GSSG (by High-Throughput Glutathione Assay kit) and 4-HNE (by OxiSelect HNE Adduct ELISA Kit) were measured in cell lysates using ELISA kits (R\&D Systems, Inc., Minneapolis, MN, USA), according to the manufacturer's protocol. Whole-cell lysates were prepared in $0.1 \%$ Nonidet 40-containing lysis buffer (Wako Pure Chemical Industries, Ltd.) as previously described (12).

Reverse transcription-polymerase chain reaction (RT-PCR). Total RNA was extracted using an RNeasy kit from $1 \times 10^{7}$ CT26 cells according to the manufacturer's protocol (Qiagen, Inc., Valencia, CA, USA). cDNA was synthesized from $0.5 \mu \mathrm{g}$ RNA using ReverTra Ace RT-qPCR kit (Toyobo Co., Ltd., Osaka, Japan) and used as a template for q-PCR. The thermocycler settings were 30 cycles of $96^{\circ} \mathrm{C}$ for $30 \mathrm{sec}, 64^{\circ} \mathrm{C}$ for $30 \mathrm{sec}$ and $72^{\circ} \mathrm{C}$ for $30 \mathrm{sec}$, followed by $72^{\circ} \mathrm{C}$ for $10 \mathrm{~min}$. The expression of monocarboxylic acid transporter (Mct)1, Mct5, succinyl-CoA transferase (Scot), NADH dehydrogenase (ubiquinone) $1 \alpha$ subcomplex, 9 (Ndufa9), succinate dehydrogenase complex, subunit A, flavoprotein (Sdha), Ubiquinol cytochrome $c$ reductase core protein 2 (Uqcrc2), cytochrome $c$ oxidase II (mtCo2), ATP synthase, $\mathrm{H}+$ transporting, mitochondrial F0 complex, subunit F2 (Atp5j2), F1 ATPase inhibitor (If1), PTEN-induced putative kinase 1 (Pink1) and GAPDH were quantified. Primers were synthesized by Sigma Genosys (Ishikari, Japan); the sequences are presented in Table I. PCR products were separated by electrophoresis in $2 \%$ agarose, and

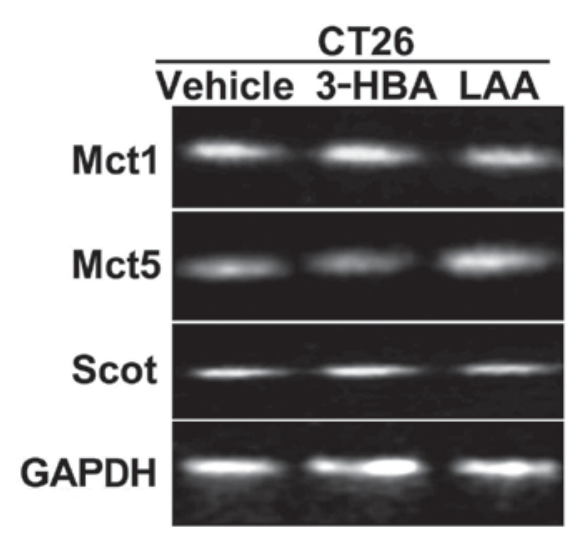

Figure 1. Expression of fatty acid transporters and a ketone body-metabolizing enzyme in CT26 cells. Expression of the monocarboxylate transporters MCT1 and MCT5 and SCOT in CT26 cells treated with 3-HBA (1 mM) or LAA $(1.5 \mathrm{mM})$ was examined by reverse transcription-polymerase chain reaction. GAPDH, loading control. SCOT, 3-oxoacid CoA transferase; MCT, monocarboxylate transporter; 3-HBA, 3-hydroxybutyric acid; LAA, lauric acid.

then stained with ethidium bromide (Wako Pure Chemical Industries, Ltd.) for $30 \mathrm{~min}$ at room temperature. The level present in untreated cells grown under $450 \mathrm{mg} / \mathrm{dl}$ glucose was set as $10 \%$. For Pink1, semi-quantitative analysis, the level present in untreated cells grown under glucose free-conditions was set as $100 \%$. Semi-quantitation was performed on captured gel images from an EPSON image scanner (Tokyo, Japan). The captured images were analyzed by densitometry with NIH Image (version 1.63; National Institute of Mental Health, Bethesda, MD, USA). Each experiment was performed in triplicate.

Statistical analysis. Statistical significance was calculated using two-tailed Fisher's exact, $\chi^{2}$ and unpaired Student t-tests using InStat software (version 3.0; GraphPad Software, Inc., La Jolla, CA, USA). Data are expressed as the mean \pm standard deviation of three independent experiments. $\mathrm{P}<0.05$ (two-sided) was considered to indicate a statistically significant difference.

\section{Results}

Expression of fatty acid transporters and ketone body metabolism-associated genes in CT26 cells. First, the mRNA expression of transporters for short-chain fatty acids (SCFAs) and MCFAs in CT26 cells treated with 3-HBA and LAA was determined (Fig. 1). 3-HBA has an aliphatic chain similar to that of SCFAs and is transported by monocarboxylate transporters (Mcts) 1-4, while LAA is an MCFA transported by Mcts 5-7 $(13,14)$. The results indicated that CT26 cells expressed Mct1 and Mct5. CT26 cells also expressed 3-oxoacid CoA transferase (SCOT), a key enzyme involved in metabolizing 3-HBA to acetoacetate $(15,16)$; however, mRNA levels were low and not significantly affected by 3 -HBA or LAA treatment (Fig. 1).

Effect of 3-HBA and LAA on CT26 cell proliferation, stemness and oxidative stress. The effect of 3-HBA and LAA on CT26 cells grown in the presence of various glucose concentrations 


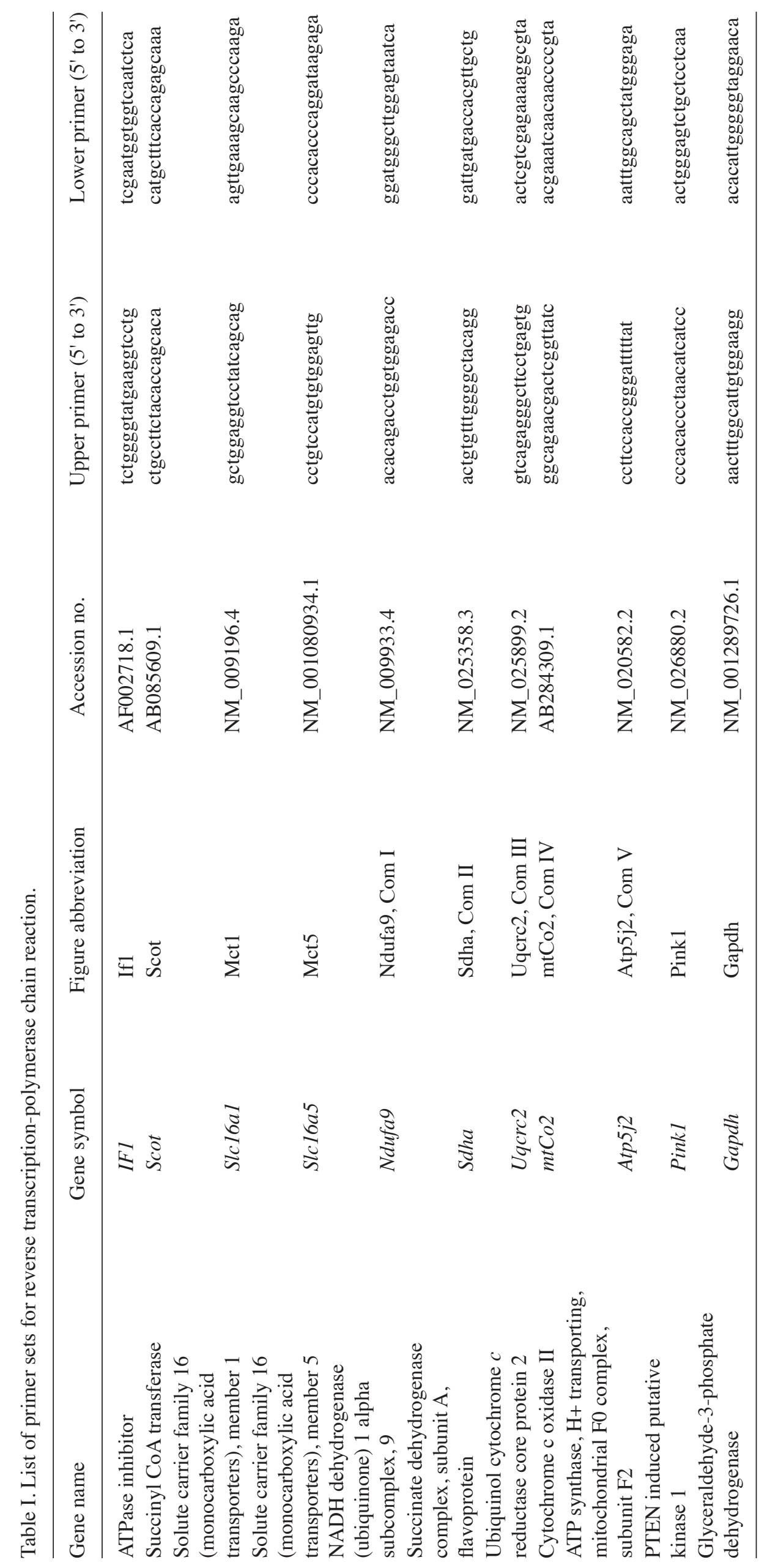



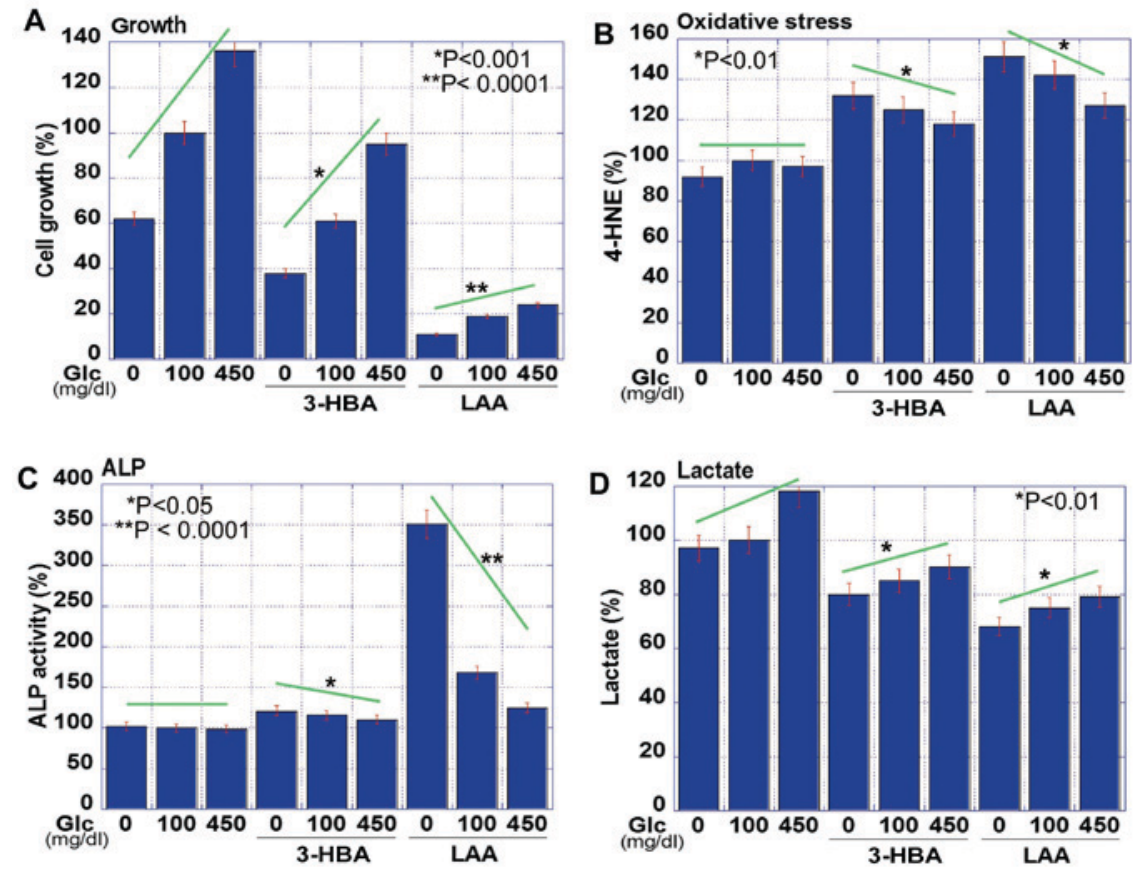

Figure 2. Effect of 3-HBA and LAA in CT26 cells. (A) Cell growth, (B) oxidative stress (4-HNE), (C) cell stemness (ALP) and (D) non-mitochondrial energy production (lactate) were examined in CT26 cells grown under various concentrations of glucose and treated with 3-HBA or LAA. The data are expressed as the mean \pm standard deviation of three independent experiments. 3-HBA, 3-hydroxybutyric acid; LAA, lauric acid; ALP, alkaline phosphatase; Glc, glucose; 4-HNE, 4-hydroxynonenal.

was subsequently examined (Fig. 2). The results revealed that the proliferation of CT26 cells was correspondingly increased with the glucose concentration, but the addition of 3-HBA or LAA into the medium inhibited cell proliferation at each glucose concentration (Fig. 2A). The suppressive effect of LAA on cell growth was markedly high. Lipid peroxidation assessed based on 4-HNE levels was not significantly different in any glucose concentration. By contrast, 4-HNE levels were upregulated with 3-HBA, and more so with LAA (Fig. 2B). Cancer cell stemness was evaluated via ALP activity (17), which was inversely associated with the glucose concentration (Fig. 2C). The 3-HBA increased ALP activity in glucose-free, but not in glucose-supplemented, medium. By contrast, LAA induced ALP activity under all conditions. Lactate concentration in the medium increased with the glucose concentration, but was decreased following the administration of 3-HBA and LAA (Fig. 2D).

Effect of 3-HBA and LAA on mitochondrial volume in CT26 cells. The effect of 3-HBA and LAA on mitochondrial volume in CT26 cells was then examined, and it was revealed that it inversely associated with the glucose concentration in the cells treated with 3-HBA or LAA (Fig. 3). The 3-HBA increased mitochondrial volume at glucose concentrations of 0 and $100 \mathrm{mg} / \mathrm{dl}$ (Fig. 3A and B). By contrast, LAA decreased mitochondrial volume at glucose concentrations of 100 and $450 \mathrm{mg} / \mathrm{dl}$ (Fig. 3D-H).

Effect of concurrent LAA and 3-HBA treatment on CT26 cell proliferation, stemness and oxidative stress. The concurrent treatment with LAA and 3-HBA inhibited cell proliferation and ALP activity in CT26 cells (Fig. 4A and B) and significantly increased GSSG (Fig. 4C), whilst also decreasing lactate (Fig. 4D) under all glucose concentrations $(\mathrm{P}<0.05)$. LAA and 3-HBA decreased mitochondrial volume at all glucose concentrations, compared with that at $100 \mathrm{mg} / \mathrm{ml}$ glucose alone; however, higher glucose concentrations induced a more pronounced decrease in mitochondrial volume (Fig. 4E-G).

Effect of 3-HBA and LAA on the expression of enzyme complexes involved in oxidative phosphorylation. The increase in oxidative stress and alterations to mitochondrial volume indicated that 3-HBA and LAA may affect oxidative phosphorylation in mitochondria. Therefore, the mRNA expression of enzymatic complexes in the oxidative phosphorylation system was examined by RT-PCR (Fig. 5). Semi-quantitative analysis of the results revealed that the expression of electron transfer system complexes in the mitochondria was altered by glucose concentration, 3-HBA and/or LAA treatments in comparison with those in glucose-free medium (Fig. 5B). In detail, treatment with 3-HBA decreased the levels of complex V under glucose-free and normal $(100 \mathrm{mg} / \mathrm{dl})$ glucose concentrations, whereas LAA administered alone downregulated the expression of all complexes in glucose-free conditions, and when in combination with 3-HBA reduced the expression of complexes under glucose-free as well as high-glucose conditions (Fig. 5B).

Furthermore, gene expression of the mitochondrial volume-associated factors inhibitor factor 1 (Ifl) and protein phosphatase and tensin homolog-induced kinase 1 (Pinkl) was examined (Fig. 5C and D). In the untreated control cells, IF1 expression was not detected, irrespective of the glucose concentration; however, 3-HBA and LAA upregulated If 1 mRNA to detectable levels in each condition, and 

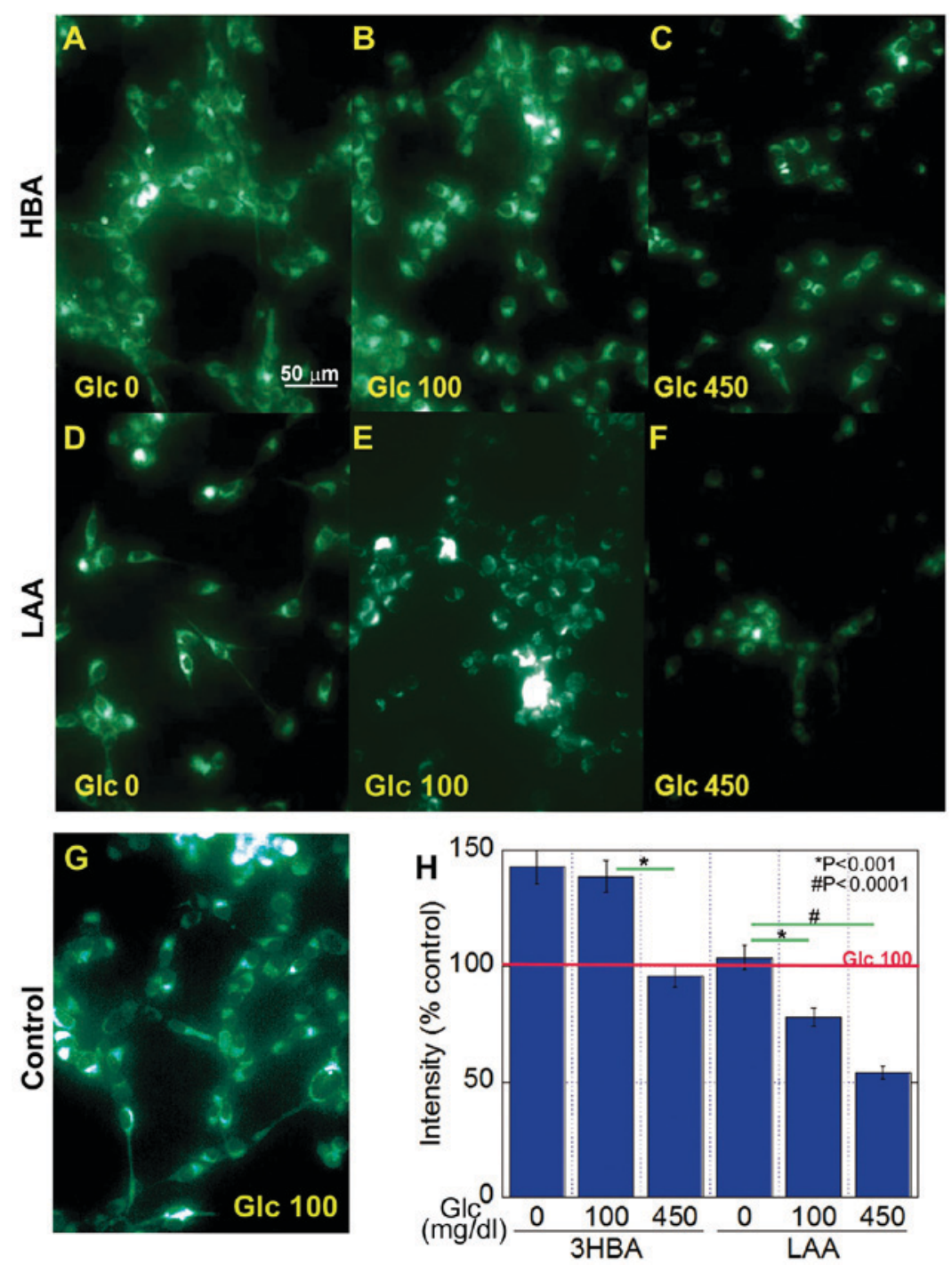

Figure 3. Effect of 3-HBA or LAA on mitochondrial volume in CT26 cells. Mitochondrial volume was examined in CT26 cells treated with 3-HBA (A-C) or LAA (D-F) using MitoGreen. Each panel is representative of the following treatments: (A) Glucose-free with $1 \mathrm{mM} 3$-HBA; (B) $100 \mathrm{mg} / \mathrm{dl}$ glucose with $1 \mathrm{mM}$ 3-HBA; (C) $450 \mathrm{mg} / \mathrm{dl}$ glucose with $1 \mathrm{mM}$ 3-HBA; (D) Glucose-free with $1.5 \mathrm{mM} \mathrm{LAA}$; (E) $100 \mathrm{mg} / \mathrm{dl}$ glucose with $1.5 \mathrm{mM}$ LAA; (F) $450 \mathrm{mg} / \mathrm{dl}$ glucose with $1.5 \mathrm{mM} \mathrm{LAA}$; (G) $100 \mathrm{mg} / \mathrm{dl}$ glucose without LAA (control). (H) Fluorescence intensity was quantified relative to the control, which was set as $100 \%$. The data are expressed as the mean \pm standard deviation of three independent experiments. 3-HBA, 3-hydroxybutyric acid; LAA, lauric acid; Glc, glucose.

the concurrent treatment of 3-HBA with LAA markedly increased IF1 expression. Pinkl expression was constitutive in control cells, irrespective of the glucose concentration. The 3-HBA treatment did not significantly affect Pinkl levels; however, they were decreased by treatment with LAA and with $3-\mathrm{HBA}+$ LAA, particularly in cells cultured at high glucose concentrations.

\section{Discussion}

In the present study, it was identified that the addition of 3-HBA or LAA inhibited the growth of CT26 colon cancer cells. In normal tissues, 3-HBA and LAA are utilized for metabolic processes, including energy production and lipogenesis, particularly in hypoglycemic conditions (13-16). The MCT isoforms 1-4 are transporters of SCFAs and monocarboxylates, including 3-HBA (13). It has been demonstrated that, in a hypermetabolic state, MCTs 1-4 are upregulated in the brain in order to transport ketone bodies and lactate (14). MCFAs, however, are transported by MCTs 5-7 and, in contrast to LCFAs, do not require the carnitine shuttle to be imported into mitochondria $(6,15)$.

Cancer cells can utilize ketone bodies or LAA $(16,18)$. It was previously revealed that gastric cancer cells could continue growing in glucose-free medium supplemented with ketone bodies (16). Utilization of ketone bodies in gastric cancer cells is regulated by the expression of SCOT, a key enzyme involved in ketone body metabolism (16), and it was reported that neuroblastoma cells were unable to use ketone bodies as an energy source, due to decreased SCOT expression (18).

Therefore, the present study examined the expression of MCT1, MCT5 and SCOT, which enable the utilization of 3-HBA and LAA in colon cancer cells. However, the levels of SCOT expression were low, which is consistent with the lower SCOT levels in cancer cells compared with in cancerassociated fibroblasts observed in colorectal cancer (19). By contrast, the carnitine shuttle enzyme carnitine palmitoyltransferase 1A (CPT1), which is important for fatty acid 

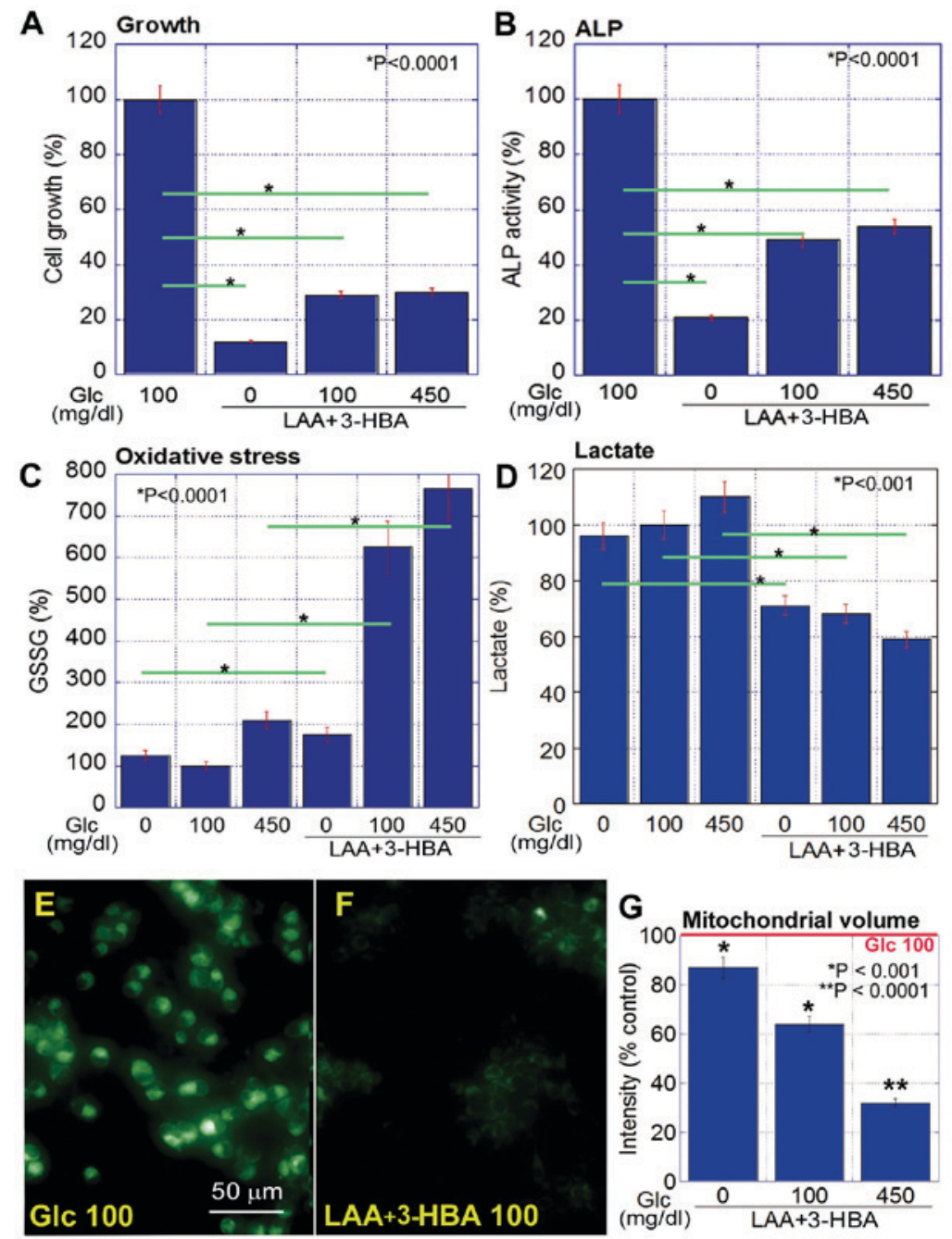

Figure 4. Effect of concurrent treatment with LAA and 3-HBA in CT26 cells. (A) Cell proliferation, (B) stemness (ALP), (C) oxidative stress (GSSG) and (D) non-mitochondrial energy production (lactate). The data are expressed as the mean $\pm \mathrm{SD}$ of three independent experiments. (E and F) Mitochondrial volume was examined in CT26 cells treated with glucose $(100 \mathrm{mg} / \mathrm{dl}$ as a control) (E) or LAA (1.5 mM) and 3-HBA (1 mM) (F) using MitoGreen. (G) Fluorescence intensity was quantified as relative to the control set as $100 \%$. The data are expressed as the mean \pm SD of three independent experiments. SD, standard deviation. 3-HBA, 3-hydroxybutyric acid; LAA, lauric acid; Glc, glucose; ALP, alkaline phosphatase; GSSG, glutathione disulfide.

transport into mitochondria, was expressed at high levels in CT26 cells (20). The experimental concentration of 3 -HBA $(1 \mathrm{mM})$ in the present study was equivalent to serum levels of ketone bodies present in individuals fed a carbohydrate-limited diet (21). The experimental concentration of LAA was also equivalent to the serum fatty acid concentration (22). Thus, LAA may provide higher mitochondria-activating effect on energy metabolism compared with 3-HBA.

In normal tissues, SCFAs and MCFAs inhibit glycolysis and stimulate lipogenesis or gluconeogenesis (15). SCFAs and MCFAs exhibit no or weak protonophoric and lytic activities in mitochondria, and decrease the efficacy of oxidative ATP synthesis (15). However, SCFAs and MCFAs also increase the mitochondrial respiratory capacity in normal physiological and inflammatory conditions (23). MCFAs also stimulate fatty acid oxidization and energy production in mitochondria in type II skeletal muscle (24). In cancer, SCOT, the rate-limiting enzyme for the production of ketone bodies, and CPT1, the carnitine shuttle protein, is associated with the cell metastatic potential, as evidenced by their increased expression in the highly metastatic SW620 cells, as compared with in the low metastatic parental SW480 cells (25). In the present study, 3-HBA and LAA decreased lactate fermentation, particularly under glucose starvation, indicating that 3-HBA and LAA switch the energy production pathway from glycolysis and lactate fermentation to oxidative phosphorylation.

Oxidative phosphorylation is considered a major intracellular source of oxidative stress (26). Activation of mitochondria is followed by oxidative stress, which may induce cell and tissue damage (26). Hydrogen peroxide release is increased in mitochondria during the oxidation of MCFAs supplied in the form of carnitine esters (27). Consistent with these findings, the present results indicated that 3-HBA and LAA increased oxidative stress in parallel with the enhancement of energy production in mitochondria.

The imbalance in the complexes of the electron transport chain (ETC), characteristic of aged mitochondria, induces the generation of ROS (28). Notably, 3-HBA and LAA caused the imbalance of ETC complexes in addition to the downregulation of PINK1, which is a major factor in PINK1-Parkin-mediated mitophagy (29). The decrease in PINK1 suggests retardation of the mitochondrial turnover, which may promote the imbalance 

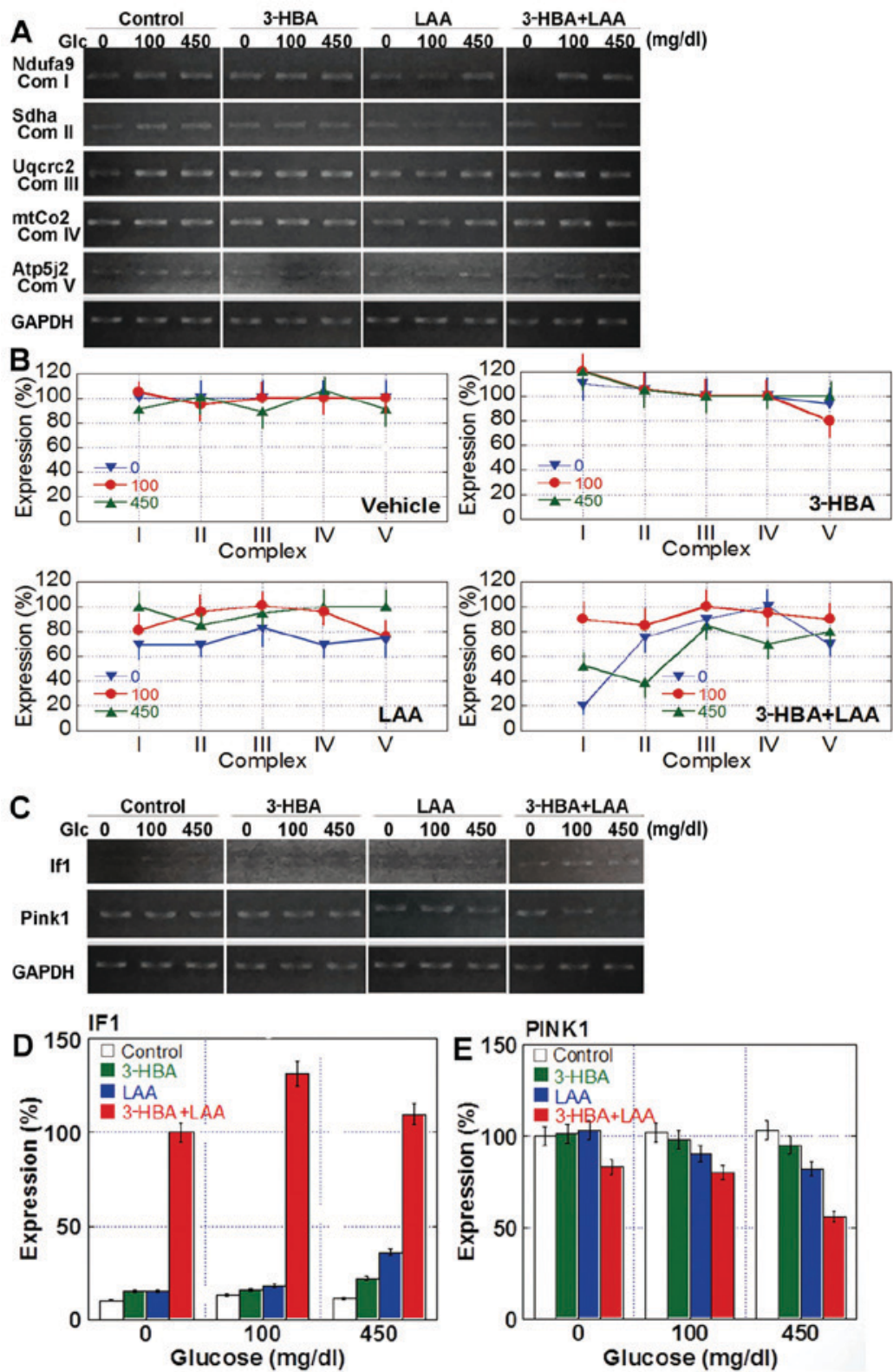

Figure 5. Expression of mitochondrial genes in CT26 cells treated with 3-HBA and/or LAA. (A) Expression of the Ndufa9 (complex I), Sdha (complex II), Uqcrc2 (complex III), $m t C o 2$ (complex IV) and Atp5j2 (complex V) genes were examined by RT-PCR. GAPDH, loading control. (B) Semi-quantitative analysis of the expression of mitochondrial complex enzymes. (C) Expression of the Ifl-and Pinkl-encoding genes was examined by RT-PCR. GAPDH, loading control. (D) Semi-quantitative analysis of IF1 expression. The level present in untreated cells grown under $450 \mathrm{mg} / \mathrm{dl}$ glucose was set as $10 \%$. (E) Semi-quantitative analysis of Pink1 expression. The level present in untreated cells grown under glucose free-conditions was set as $100 \%$. The data are expressed as the mean \pm standard deviation of three independent experiments. RT-PCR, reverse transcription-polymerase chain reaction; 3-HBA, 3-hydroxybutyric acid; LAA, lauric acid; If1, F1 ATPase inhibitor; Pink1, protein phosphatase and tensin homolog-induced kinase 1; Sdha, succinate dehydrogenase complex, subunit; Uqcrc2, ubiquinol cytochrome $c$ reductase core protein 2; mtCo2, cytochrome $c$ oxidase II; Ndufa9, NADH dehydrogenase (ubiquinone) 1 $\alpha$ subcomplex, 9; Atp5j2, ATP synthase, $\mathrm{H}+$ transporting, mitochondrial F0 complex, subunit F2.

of ETC complexes and result in ROS production, which in turn may damage the mitochondria. The present data suggested that 3-HBA- and LAA-associated oxidative stress is associated with the decrease in mitochondrial volume. The depletion of proteins involved in autophagy leads to mitochondrial dysfunction, decreased ATP production and increased ROS release, ultimately resulting in the apoptosis of mesenchymal stem cells (30).

Thus, the present data suggested that ketone bodies and MCFAs may exert anticancer effects via the deviation of energy metabolism from glycolysis-lactate fermentation to oxidative phosphorylation. Additional studies are required to compare the responses to ketone bodies and MCFAs in normal and cancer tissues, in order to verify the therapeutic effect of LAA and 3-HBA dietary supplementation for patients with cancer.

\section{Acknowledgements}

The authors thank Ms. Tomomi Masutani for expert assistance with the preparation of this manuscript. The present study was supported by Mext Kakenhi (grant nos. 14478268 and 16675788). 


\section{References}

1. Chen X, Qian Y and Wu S: The Warburg effect: Evolving interpretations of an established concept. Free Radic Biol Med 79: 253-263, 2015

2. Warburg O: On respiratory impairment in cancer cells. Science 124: 269-270, 1956.

3. Hess JA and Khasawneh MK: Cancer metabolism and oxidative stress: Insights into carcinogenesis and chemotherapy via the non-dihydrofolate reductase effects of methotrexate. BBA Clin 3: 152-161, 2015.

4. Boland ML, Chourasia AH and Macleod KF: Mitochondrial dysfunction in cancer. Front Oncol 3: 292, 2013.

5. Dueregger A, Schöpf B, Eder T, Höfer J, Gnaiger E, Aufinger A, Kenner L, Perktold B, Ramoner R, Klocker H and Eder IE: Differential utilization of dietary fatty acids in benign and malignant cells of the prostate. PLoS One 10: e0135704, 2015.

6. Papamandjaris AA, MacDougall DE and Jones PJ: Medium chain fatty acid metabolism and energy expenditure: Obesity treatment implications. Life Sci 62: 1203-1215, 1998.

7. Newman JC and Verdin E: $\beta$-hydroxybutyrate: Much more than a metabolite. Diabetes Res Clin Pract 106: 173-181, 2014

8. Fukao T, Mitchell G, Sass JO, Hori T, Orii K and Aoyama Y: Ketone body metabolism and its defects. J Inherit Metab Dis 37 541-551, 2014

9. McCarty MF and DiNicolantonio JJ: Lauric acid-rich medium-chain triglycerides can substitute for other oils in cooking applications and may have limited pathogenicity. Open Heart 3: e000467, 2016.

10. Fulgencio JP, Kohl C, Girard J and Pégorier JP: Troglitazone inhibits fatty acid oxidation and esterification, and gluconeogenesis in isolated hepatocytes from starved rats. Diabetes 45: 1556-1562, 1996.

11. Kuniyasu H, Yano S, Sasaki T, Sasahira T, Sone S and Ohmori H: Colon cancer cell-derived high mobility group 1/amphoterin induces growth inhibition and apoptosis in macrophages. Am J Pathol 166: 751-760, 2005.

12. Kuniyasu H, Oue N, Wakikawa A, Shigeishi H, Matsutani N, Kuraoka K, Ito R, Yokozaki $\mathrm{H}$ and Yasui W: Expression of receptors for advanced glycation end-products (RAGE) is closely associated with the invasive and metastatic activity of gastric cancer. J Pathol 196: 163-170, 2002.

13. Schutkowski A, Wege N, Stangl GI and König B: Tissue-specific expression of monocarboxylate transporters during fasting in mice. PLoS One 9: e112118, 2014.

14. Takimoto $M$ and Hamada T: Acute exercise increases brain region-specific expression of MCT1, MCT2, MCT4, GLUT1, and COX IV proteins. J Appl Physiol (1985) 116: 1238-1250, 2014.

15. Schönfeld P and Wojtczak L: Short- and medium-chain fatty acids in energy metabolism: The cellular perspective. J Lipid Res 57: 943-954, 2016.

16. Sawai M, Yashiro M, Nishiguchi Y, Ohira M and Hirakawa K: Growth-inhibitory effects of the ketone body, monoacetoacetin, on human gastric cancer cells with succinyl-CoA: 3-oxoacid CoA-transferase (SCOT) deficiency. Anticancer Res 24: 2213-2217, 2004

17. Stefková K, Procházková J and Pacherník J: Alkaline Phosphatase in Stem Cells. Stem Cell Int 2015: 628368, 2015.
18. Skinner R, Trujillo A, Ma X and Beierle EA: Ketone bodies inhibit the viability of human neuroblastoma cells. J Pediatr Surg 44: 212-216, 2009.

19. Chao C, Carmical JR, Ives KL, Wood TG, Aronson JF, Gomez GA, Djukom CD and Hellmich MR: CD133+ colon cancer cells are more interactive with the tumor microenvironment than CD133- cells. Lab Invest 92: 420-436, 2012.

20. Park JH, Vithayathil S, Kumar S, Sung PL, Dobrolecki LE, Putluri V, Bhat VB, Bhowmik SK, Gupta V, Arora K, et al: Fatty acid oxidation-driven src links mitochondrial energy reprogramming and oncogenic properties in triple-negative breast cancer. Cell Rep 14: 2154-2165, 2016.

21. Carneiro L, Geller S, Fioramonti X, Hébert A, Repond C, Leloup $\mathrm{C}$ and Pellerin L: Evidence for hypothalamic ketone body sensing: Impact on food intake and peripheral metabolic responses in mice. Am J Physiol Endocrinol Metab 310: E103-E115, 2016.

22. Allen BG, Bhatia SK, Anderson CM, Eichenberger-Gilmore JM, Sibenaller ZA, Mapuskar KA, Schoenfeld JD, Buatti JM, Spitz DR and Fath MA: Ketogenic diets as an adjuvant cancer therapy: History and potential mechanism. Redox Biol 2: 963-970, 2014.

23. Hecker M, Sommer N, Voigtmann H, Pak O, Mohr A, Wolf M, Vadász I, Herold S, Weissmann N, Morty RE, et al: Impact of short- and medium-chain fatty acids on mitochondrial function in severe inflammation. JPEN J Parenter Enteral Nutr 38: 587-594, 2014.

24. Ishizawa R, Masuda K, Sakata S and Nakatani A: Effects of different fatty acid chain lengths on fatty acid oxidation-related protein expression levels in rat skeletal muscles. J Oleo Sci 64: 415-421, 2015.

25. Provenzani A, Fronza R, Loreni F, Pascale A, Amadio M and Quattrone A: Global alterations in mRNA polysomal recruitment in a cell model of colorectal cancer progression to metastasis. Carcinogenesis 27: 1323-1333, 2006.

26. Chen Y, Zhang H, Zhou HJ, Ji W and Min W: Mitochondrial redox signaling and tumor progression. Cancers (Basel) 8: pii: E40, 2016.

27. Schonfeld $P$ and Wojtczak L: Brown adipose tissue mitochondria oxidizing fatty acids generate high levels of reactive oxygen species irrespective of the uncoupling protein-1 activity state. Biochim Biophys Acta 1817: 410-418, 2012.

28. Lee YK, Jee BA, Kwon SM, Yoon YS, Xu WG, Wang HJ, Wang XW, Thorgeirsson SS, Lee JS, Woo HG and Yoon G: Identification of a mitochondrial defect gene signature reveals NUPR1 as a key regulator of liver cancer progression. Hepatology 62: 1174-1189, 2015.

29. Ploumi C, Daskalaki I and Tavernarakis N: Mitochondrial biogenesis and clearance: A balancing act. FEBS J 284: 183-195, 2017.

30. Ghanta S, Tsoyi K, Liu X, Nakahira K, Ith B, Coronata AA, Fredenburgh LE, Englert JA, Piantadosi CA, Choi AM and Perrella MA: Mesenchymal stromal cells deficient in autophagy proteins are susceptible to oxidative injury and mitochondrial dysfunction. Am J Respir Cell Mol Biol 56: 300-309, 2016. 